\title{
Space-charge limited surface currents between two semi-infinite planar electrodes embedded in a uniform dielectric medium
}

\author{
P. De Visschere ${ }^{\mathrm{a}, \mathrm{b}, *}$, W. Woestenborghs ${ }^{\mathrm{a}, \mathrm{b}}, \mathrm{K}$. Neyts $^{\mathrm{a}, \mathrm{b}}$ \\ ${ }^{a}$ Liquid Crystals 83 Photonics Group, Ghent University, Sint-Pietersnieuwstraat 41, 9000 \\ Ghent, Belgium \\ ${ }^{b}$ Center for Nano and Biophotonics, Ghent University
}

\begin{abstract}
We extend the one-dimensional space-charge limited current theory to a twodimensional geometry where current flows in a thin layer between two coplanar semi-infinite electrodes. It is shown that the surface charge density in the gap between the electrodes is the finite Hilbert transform of the in-plane component of the electric field. This enables us to derive analytical expressions for the field and charge density for single carrier injection and for photo-carrier extraction by solving a non-linear integral equation for the field. The analytical expressions have been verified by numerical calculations. For the in-plane geometry, the one-dimensional Mott-Gurney equation $J=\frac{9}{8} \mu \epsilon \frac{V^{2}}{L^{3}}$ is replaced by a similar $K=\frac{2}{\pi} \mu \epsilon \frac{V^{2}}{L^{2}}$ equation. For extraction of photo-generated carriers the one-dimensional $J \sim g^{3 / 4} V^{1 / 2}$ dependence is replaced by a $K \sim g^{2 / 3} V^{2 / 3}$ dependence, where $g$ is the generation rate of photo-carriers. We also extend these results to take into account trapping. We show experimental evidence obtained with an organic photoconductor confirming the predicted voltage, width and generation dependencies.
\end{abstract}

\section{Introduction}

Space-charge limited currents have always played a pertinent role in electronic devices, starting with the vacuum tube [1,2], subsequently in solid-state electronic devices [3] and more recently in organic electronic devices [4, 5]. In a vacuum tube the space-charge limited electron current is found based on energy conservation and Poisson's equation and leads to the Child-Langmuir equation

$$
J=\frac{4}{9} \sqrt{\frac{2 e}{m}} \epsilon_{0} \frac{V^{3 / 2}}{L^{2}}
$$

\footnotetext{
*Corresponding author

Email address: pdv@elis.ugent.be (P. De Visschere)
} 
with $e$ and $m$ the electron charge and mass, $\epsilon_{0}$ the dielectric constant of vacuum and $L$ the gap between the parallel electrodes and $V$ the applied voltage. If only a single type of carrier is injected in an insulator without traps, a similar theory leads to the Mott-Gurney equation

$$
J=\frac{9}{8} \mu \epsilon \frac{V^{2}}{L^{3}}
$$

where $\mu$ is the mobility of the carrier and $\epsilon$ the dielectric constant. If a photoconductor with non-injecting contacts shows a large asymmetry in the mobilities of electrons and holes then a space-charge develops mainly near one electrode when extracting the photo-carriers by applying a bias voltage. In the past one has applied (2) to this space-charge region [6] but a more precise calculation (mentioned later) yields a numerical factor 4 instead of $9 / 8$

$$
J \approx e g W=4 \mu \epsilon \frac{V^{2}}{W^{3}}
$$

where $g$ is the volume generation rate of photo-carriers and $W$ the width of the space-charge layer. The difference stems from the hole and electron currents not being constant in the space-charge layer. Eliminating $W$ from (3) yields

$$
J \approx(4 \mu \epsilon)^{1 / 4}(e g)^{3 / 4} \sqrt{V}
$$

with a square root dependence on the voltage and a $g^{3 / 4}$ dependence on the irradiance.

If $W=L$ then the current saturates and this occurs for a voltage

$$
V_{\mathrm{sat}}=\frac{1}{2} \sqrt{\frac{e g}{\mu \epsilon}} L^{2}
$$

These are the simplest models known for space-charge limited currents but modeling real devices usually becomes more complicated because e.g. both types of carriers are injected, or because traps are present $[3,7,8]$ or because the mobility is field dependent [9] and/or carrier density dependent [10] and so on.

All formulas mentioned and possible extensions have been derived for a planar one-dimensional structure. Langmuir [2] also considered cylindrical electrodes, and noted that the $V^{3 / 2}$ dependence in (1) does not depend on the shape of the electrodes, using a scaling argument. Only recently the Child-Langmuir law was extended to electron emission over a finite patch on a planar cathode $[11,12,13]$.

Whereas the geometry of many practical devices is indeed one-dimensional, there are exceptions, as just mentioned. Another example are photoconductors, which often have an in-plane geometry with interdigitated electrodes. With inorganic photoconductors usually no space-charge limitation occurs due to the relatively high mobility-lifetime product [14], but with organic photoconductors [15, 16] space-charge limited currents have been reported several times [17, 18, 19, 20]. 
In this paper we extend the theory of space-charge limited currents to the geometry of two semi-infinite coplanar electrodes, where the current flows in an infinitesimally thin channel between the electrodes, where it will be assumed that the structure is embedded in a uniform dielectric medium. This is applicable to the mentioned organic photoconductors that use finger electrodes, assuming the width of the fingers is much larger than the gap width and the structure is sealed between two glass plates. In this case the current flow is confined to the channel and is still one-dimensional but the electric field in the channel and in the dielectric medium is two-dimensional. Thin film transistors (TFTs) and in particular OTFTs have a very similar geometry but due to the extra gate electrode the field can be calculated approximately using the well-known gradual channel approximation [21]. This holds also for the photoconductive structures reported by Lombardo e.a. [22] and Ooi e.a. [23].

The rest of the paper is organized as follows. In section 2 we calculate the electric field using a conformal transformation. In the next sections this result is combined with the drift and continuity equations and the overall problem is reduced to solving a non-linear integral equation for the electric field in the gap. In section 3 we consider single carrier injection and in section 4 photocarrier extraction. The details of the calculations are given in the appendix. In section 5 some experimental evidence is presented for the theory.

\section{Two-dimensional electrostatic problem}

The electrostatic problem to be solved consists of two semi-infinite coplanar electrodes, with an applied potential difference $V$, and separated by a gap with width $L$. In the plane between the electrodes an unknown surface charge density $\rho\left[\mathrm{C} / \mathrm{m}^{2}\right]$ is present and the structure is embedded in a uniform medium with dielectric constant $\epsilon$. Since only one length parameter is involved we normalize the width of the gap with $L / 2$ and choose a coordinate system as shown in figure 1 , with the anode $-\infty<x<-1$, the cathode $+1<x<+\infty$ and the thin conducting layer $-1<x<+1$. Likewise the potential is normalized with $V$ and with these conventions the electric field and the surface charge density are both normalized with $2 V / L$. The field is split into a contribution due to the applied voltage without space-charge being present and the contribution of the space-charge density $\rho(x)$ with no voltage difference applied between the electrodes $E(x)=E_{a}(x)+E_{\rho}(x)$. For the first problem the Laplace equation must be solved in the whole 2D-plane except for two cuts along the electrodes and this problem can be solved by transforming this region into the upper halve plane using a complex Schwarz-Christoffel transformation [24, 25, 26]

$$
w=z+\sqrt{z^{2}-1}
$$

where $z=x+j y$ and $w=u+j v$ and with $0<\arg (z-1)<2 \pi$ and $-\pi<$ $\arg (z+1)<\pi$. In the transformed $w$-plane the complex potential is easily 

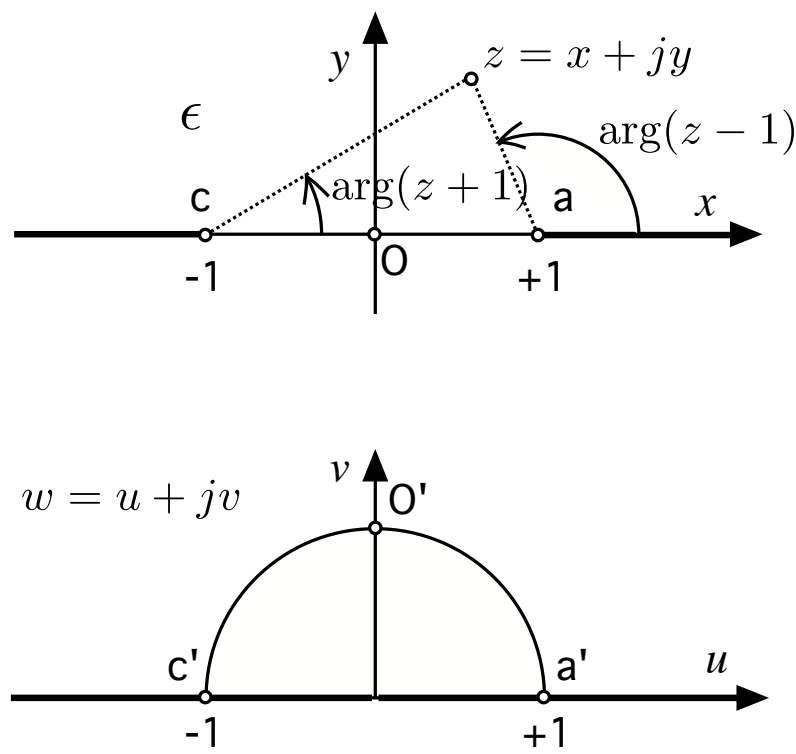

Figure 1: (top) Geometry used for solving the electrostatic problem. (bottom) The conformally transformed geometry, with the anode $-\infty<u<0$ and the cathode $0<u<+\infty$. The gap cOa is transformed into the semicircle c'O'a'.

found as $W_{a}(w)=-j \frac{1}{\pi} \ln w$ and in the original $z$-plane the complex potential is then given by

$$
W_{a}(z)=-j \frac{1}{\pi} \ln \left(z+\sqrt{z^{2}-1}\right)
$$

The $x$-component of the (applied) electric field in the gap is obtained as

$$
E_{a}(x)=-\Re\left(\frac{d W_{a}}{d z}\right)=\frac{1}{\pi} \frac{1}{\sqrt{1-x^{2}}}
$$

To obtain $E_{\rho}(x)$ we consider a line charge $q$ at an arbitrary position $z_{0}=x_{0}+j y_{0}$ and apply the same conformal transformation. In the $w$-plane the complex potential can be found as that of a line charge $q$ at $w_{0}=u_{0}+j v_{0}$ and an image-charge $-q$ at the position $w_{0}^{*}=u_{0}-j v_{0}$

$$
W_{q}(w)=-\frac{q}{2 \pi \epsilon} \ln \frac{w-w_{0}}{w-w_{0}^{*}}
$$

Using (6), taking the derivative and the negative real part, taking the limits $y, y_{0} \rightarrow 0$ and applying superposition we obtain the 2 nd contribution to the $x$-component of the field as

$$
E_{\rho}(x)=-\frac{1}{2 \pi \epsilon} \int_{-1}^{1} \sqrt{\frac{1-x^{\prime 2}}{1-x^{2}}} \frac{\rho\left(x^{\prime}\right)}{x^{\prime}-x} d x^{\prime}
$$


where the integral and all subsequent similar integrals are Cauchy principal value integrals. The integral transform on the rhs is the inverse of the finite Hilbert transform [27] and we conclude that the charge density can be calculated as the finite Hilbert transform of the electric field

$$
\frac{\rho(x)}{2 \epsilon}=\mathcal{H}(E)=\frac{1}{\pi} \int_{-1}^{1} \frac{E\left(x^{\prime}\right)}{x^{\prime}-x} d x^{\prime}
$$

Since $\mathcal{H}\left(1 / \sqrt{1-x^{2}}\right)=0$ (within the gap) we have replaced $E_{\rho}$ by the total field $E$ in (11). As an application of this relation we find the (normalized) charge density required to obtain a uniform electric field

$$
\frac{\rho(x)}{2 \epsilon}=\frac{1}{2 \pi} \ln \frac{1-x}{1+x}
$$

\section{3. space-charge limited injection current}

We consider the injection of one type of carriers, e.g. holes from an ideal reservoir into an insulator without traps. Neglecting diffusion currents, the drift equation is given by

$$
K_{\mathrm{inj}}=e p \mu_{p} E=\mu_{p} \rho E
$$

where $K_{\text {inj }}$ is the constant surface current density, $p$ the surface carrier density and $\rho$ the surface charge density. Using (11) (and taking into account the normalization of the field) we obtain a non-linear integral equation for the electric field

$$
K_{\mathrm{inj}}=\frac{2}{\pi} \epsilon \mu_{p} \frac{V^{2}}{L^{2}} 4 E(x) \int_{-1}^{1} \frac{E\left(x^{\prime}\right)}{x^{\prime}-x} d x^{\prime}
$$

which must be solved with the boundary condition $E(-1)=0$, since the anode is an ideal reservoir, and with $\int_{-1}^{1} E(x) d x=1$. Apparently the unique normalized electric field profile is found by solving

$$
E(x) \int_{-1}^{1} \frac{E\left(x^{\prime}\right)}{x^{\prime}-x} d x^{\prime}=\frac{\alpha}{4}
$$

where $K_{\text {inj }}=\frac{2}{\pi} \alpha \epsilon \mu_{p} \frac{V^{2}}{L^{2}}$. The detailed solution is given in the appendix. The constant $\alpha$ can be found by integrating this equation over the total width but since the field is singular for $x=1$, we multiply both sides with $(1-x)$ before integration

$$
\int_{-1}^{1}(1-x) E(x)\left[\int_{-1}^{1} \frac{E\left(x^{\prime}\right)}{x^{\prime}-x} d x^{\prime}\right] d x=\frac{\alpha}{2}
$$

After reversing the order of integration one can remove the singularity and then finds

$$
\alpha=\left[\int_{-1}^{1} E(x) d x\right]^{2}=1
$$


and the $K(V)$-characteristic becomes

$$
K_{\mathrm{inj}}=\frac{2}{\pi} \epsilon \mu_{p} \frac{V^{2}}{L^{2}}
$$

Apart from the different width dependence, the factor $9 / 8$ in the 1D-SCL current (2) is replaced by the factor $2 / \pi$ in the $2 \mathrm{D}$-SCL current.

To check the analytical solutions given in the appendix for the electric field and for the charge density we compare (A.3)(A.4) and (A.7) with the results of a numerical model, which uses (10) for calculating the electric field but takes into account drift and diffusion [28] (see figure 2). Boundary conditions for the numerical model were chosen to obtain conditions of single carrier injection: we used a Poole-Frenkel field emission formula for both carriers and adapted the barriers so that only holes are injected. Except for transition zones near the electrodes, the numerical results match very well with the analytical formulas. Note that the electric field has been multiplied with $\sqrt{1-x^{2}}$ to remove the singularities. Whereas the theoretical electric field has no singularity at the anode $(x=-1)$, but behaves as $E(x) \approx \frac{\left(\ln \frac{2}{1+x}-1\right)^{-1 / 2}}{2 \sqrt{2}}$, the numerically calculated field diverges. However for calculating the field emission current the numerical model averages the electric field over a width comparable with the thickness of the electrodes, so that the emission current does not diverge. Near the anode the charge density diverges according to $\frac{\rho(x)}{2 \epsilon} \approx \frac{\left(\ln \frac{2}{1+x}-1\right)^{1 / 2}}{\pi \sqrt{2}}$, which is a modest singularity. Near the cathode the electric field has a singularity $E(x) \approx \frac{1}{\pi \sqrt{1-x}}$ and the charge density behaves as a square root $\frac{\rho(x)}{2 \epsilon} \approx \frac{\sqrt{1-x}}{4}$. Figure 3 shows that for a sufficiently high voltage the numerically calculated current tends to the analytical $K(V)$ characteristic (17).

\section{4. space-charge limited photo generated current}

Next we consider an asymmetric photoconductor with e.g. $\mu_{n} \ll \mu_{p}$ and with non-injecting contacts so that a space-charge develops near the anode when extracting the photo generated carriers. We neglect the much smaller spacecharge near the cathode and focus here on the anodic space-charge layer only. Neglecting diffusion we rewrite the drift equations in terms of the average carrier density $c=(p+n) / 2$ and the space-charge density $\rho=e(p-n)$

$$
\begin{aligned}
4 e \mu c E & =K_{\text {phot }}-\gamma K_{s} \\
2 \mu \rho E & =K_{s}-\gamma K_{\text {phot }}
\end{aligned}
$$

where $\mu=\mu_{n} \mu_{p} /\left(\mu_{n}+\mu_{p}\right) \approx \mu_{n}, \gamma=\left(\mu_{p}-\mu_{n}\right) /\left(\mu_{p}+\mu_{n}\right) \approx 1, K_{\text {phot }}=$ $e\left(K_{p}-K_{n}\right)$ is the total constant current density whereas $K_{s}=e\left(K_{p}+K_{n}\right)$, with $K_{p}>0$ and $K_{n}<0$ the flux densities of the carriers. These equations must be augmented with the continuity equations $\frac{d K_{p}}{d x}=\frac{d K_{n}}{d x}=g-r$, with 


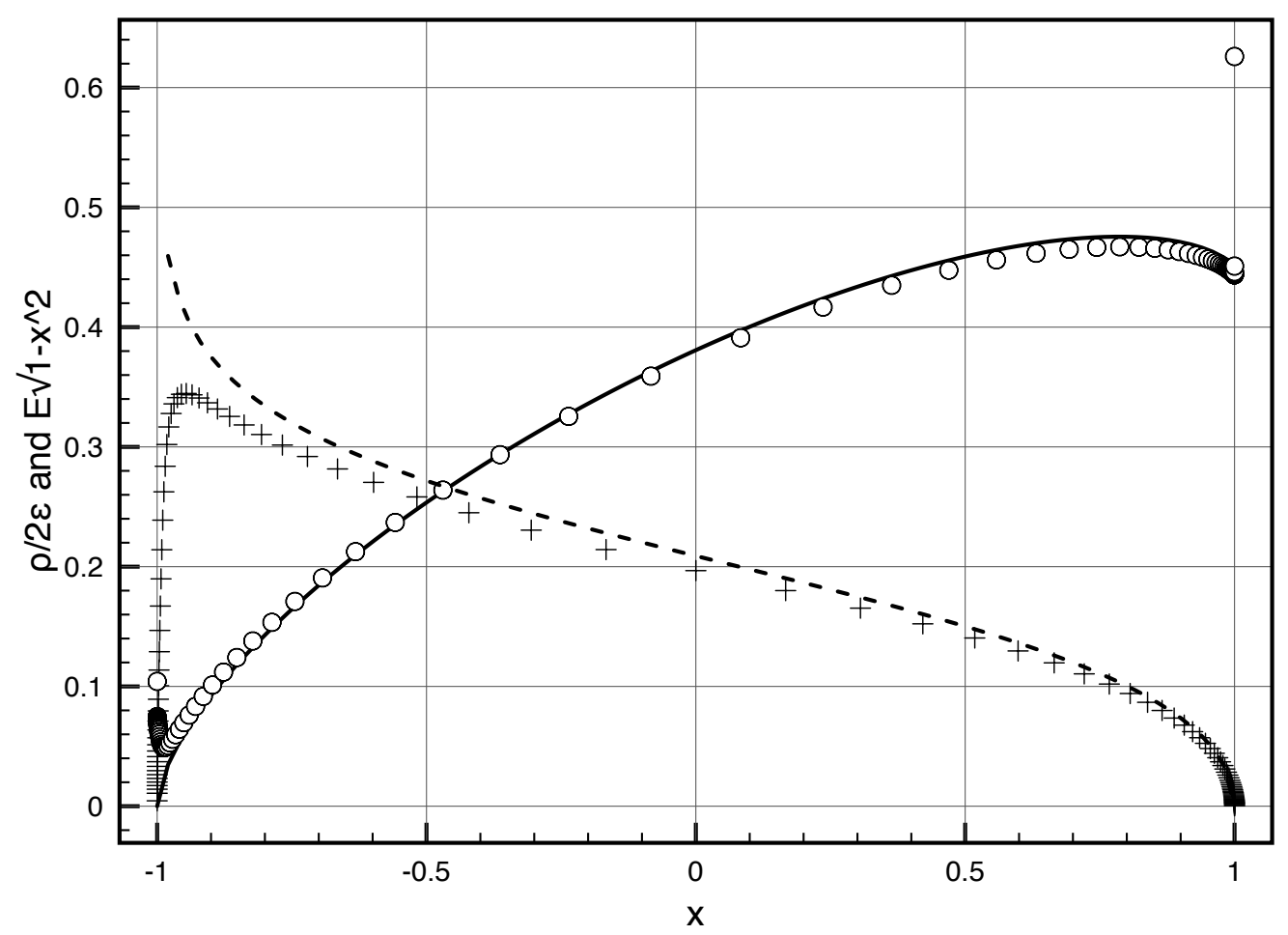

Figure 2: Comparison between the normalized electric field (open circles, full line) and charge density (plusses, broken line) for single carrier injection in an insulator at a voltage $V=100 \mathrm{~V}$, obtained with a numerical model (symbols) and with the analytical solutions given in the appendix (lines). Both quantities are normalized with $2 V / L$ and the electric field has been multiplied with $\sqrt{1-x^{2}}$ to remove the singularities. 


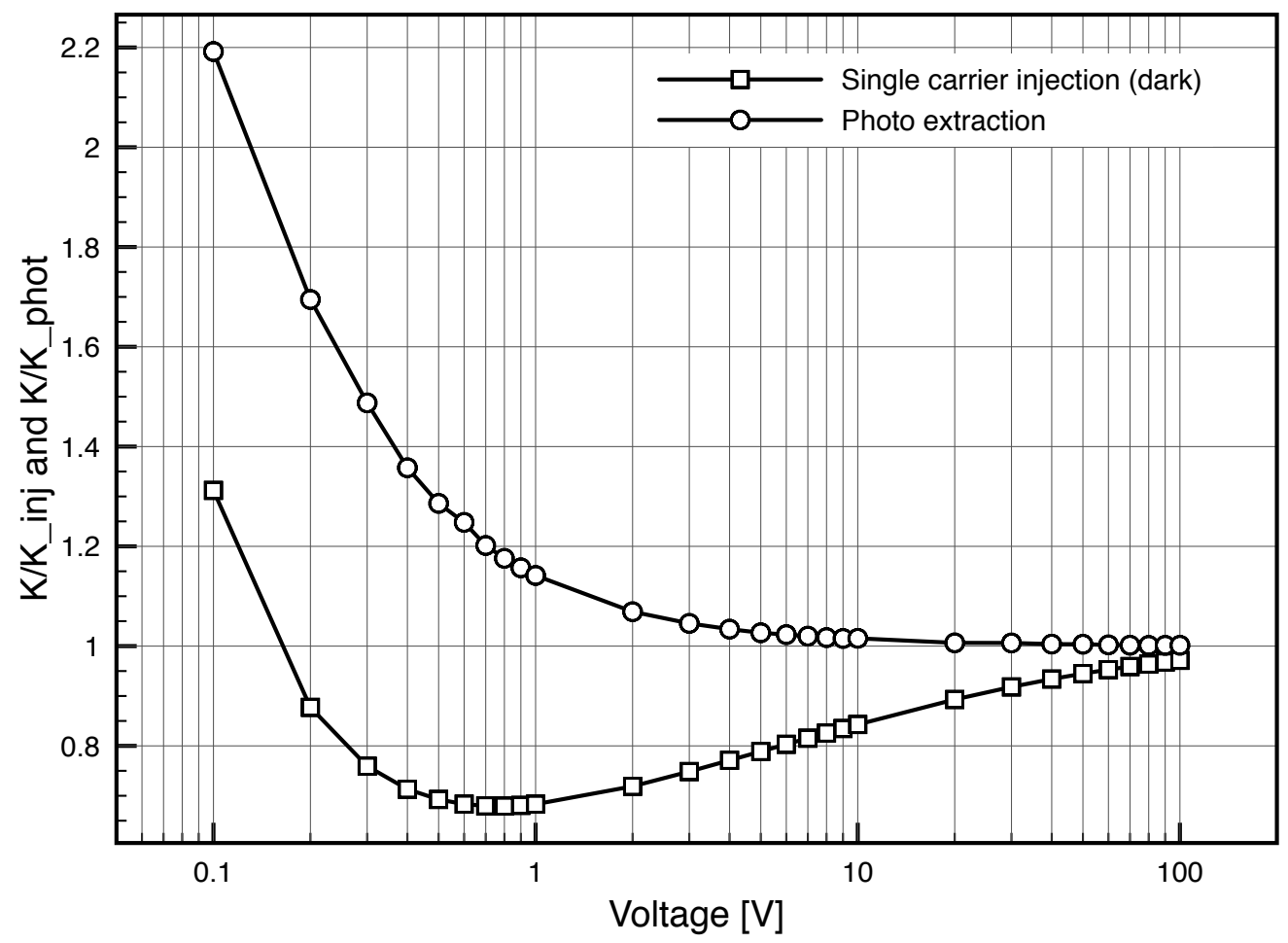

Figure 3: Ratio of the numerically calculated current density $K(V)$ over the analytical results $K_{\text {inj }}(V)$ and $K_{\text {phot }}(V)$ given by (17) and (24). 
$g$ the (surface) generation rate and $r$ the recombination rate. An approximate solution can be obtained by assuming an abrupt space-charge layer transition. Outside the space-charge layer $g \approx r$, the current densities are constant and $\rho \ll c$ so that $p \approx n$ and then the rhs of $(19)$ is zero $\left(K_{s}=\gamma K_{\text {phot }}\right)$. In the space-charge layer we neglect recombination and $\frac{d K_{s}}{d x} \approx 2 e g$. Eq. (19) can then be approximated by

$$
2 \mu \rho E=2 e g \begin{cases}\left(x-W+\frac{L}{2}\right) & -\frac{L}{2}<x<-\frac{L}{2}+W \\ 0 & -\frac{L}{2}+W<x<\frac{L}{2}\end{cases}
$$

where $W$ is the space-charge layer width. Solving this equation for a onedimensional model with $\rho=\epsilon \frac{d E}{d x}$ and neglecting the voltage drop over the bulk results into (3). For our two-dimensional model, we insert (11), we normalize the field and the geometry parameters and we take into account $K_{\text {phot }}=e g W$ and then this equation can be brought into a universal form

$$
E(x) \int_{-1}^{1} \frac{E\left(x^{\prime}\right)}{x^{\prime}-x} d x^{\prime}=\frac{\beta}{8} \begin{cases}(x-2 w+1) & -1<x<2 w-1 \\ 0 & 2 w-1<x<1\end{cases}
$$

where $w=W / L$. This equation must be solved for $\beta$ with the boundary conditions $E(2 w-1)=0$ and $\int_{-1}^{1} E(x) d x=1$. The $K(V)$-characteristic follows then as

$$
K_{\text {phot }}=\frac{2}{\pi} \beta \mu \epsilon \frac{V^{2} W}{L^{3}}
$$

The constant $\beta$ can again be found by integrating (20) over the space-charge layer width but after multiplying with $(1+x)$

$$
\int_{-1}^{1}(1+x) E(x)\left[\int_{-1}^{1} \frac{E\left(x^{\prime}\right)}{x^{\prime}-x} d x^{\prime}\right] d x=-\frac{1}{6} \beta w^{3}
$$

After reversing the order of integration the singularity can be removed and we find that

$$
\beta w^{3}=3\left[\int_{-1}^{1} E(x) d x\right]^{2}=3
$$

and the $K(V)$-characteristic becomes

$$
K_{\text {phot }}=\frac{6}{\pi} \mu \epsilon \frac{V^{2}}{W^{2}}
$$

In figure 4 a comparison is shown between the normalized electric field and the charge density calculated with (A.3)(A.4)(A.11) and (A.12) and with the numerical model mentioned in section 3. We choose a relatively high hole mobility $\mu_{p}=10^{-6} \mathrm{~m}^{2} / \mathrm{Vs}$ to limit the voltage drop outside the space-charge layer, we avoid hole injection and choose a much lower electron mobility $\mu_{n}=$ $10^{-10} \mathrm{~m}^{2} / \mathrm{Vs} \ll \mu_{p}$, so that a space-charge develops near the anode, but we allow 


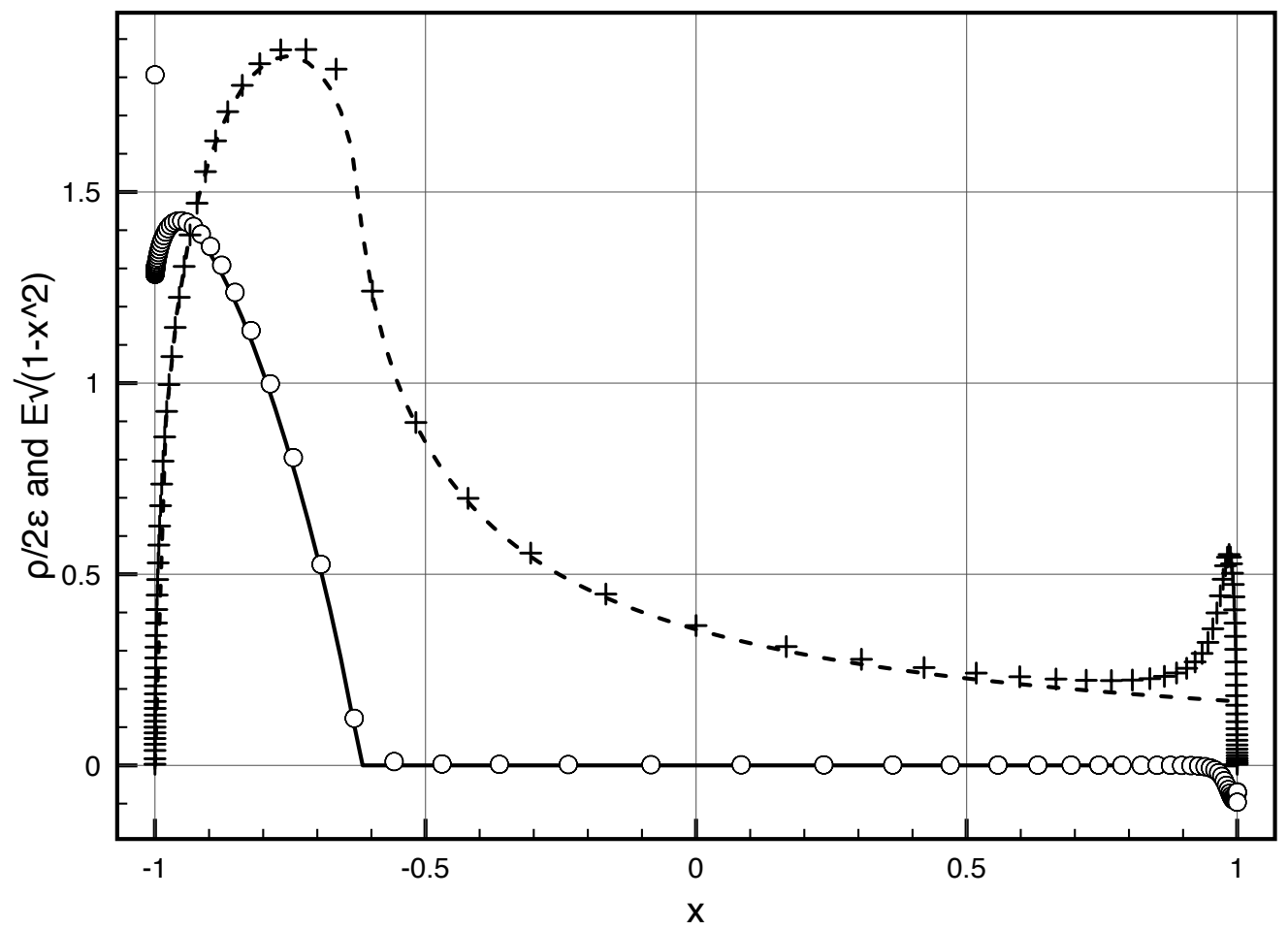

Figure 4: Comparison between the normalized electric field (open circles, full line) and charge density (plusses, dashed line) for photo-carrier extraction at a voltage $V=10 \mathrm{~V}$, obtained with a numerical model (symbols) and with the analytical solutions given in the appendix (lines). Both quantities are normalized with $2 V / L$ and the electric field has been multiplied with $\sqrt{1-x^{2}}$ to remove the singularities. From the numerical calculation we found $w=0.19$ and this was used in the analytical formulas. 
electron injection to avoid a space-charge near the cathode. The space-charge layer width $w$ needed for the analytical formulas was obtained from the numerical calculation as $w=K / e g L$, where $K$ is the current calculated numerically. Except near the cathode $(x=1)$ there is almost a perfect match between both calculations. Note that the global dependence (11) between electric field and charge density results into a smaller but non-negligible charge density outside the space-charge region. The following asymptotic dependencies can be derived from the analytical formulas: near the anode $(x=-1)$ the electric field diverges as $E(x) \approx \frac{\sqrt{3 / 2 w}}{\pi \sqrt{1+x}}$, whereas the charge density follows the inverse square root dependence $\frac{\rho(x)}{2 \epsilon} \approx-\frac{1}{2} \sqrt{3 / 2 w^{3}} \sqrt{1+x}$. Near the edge of the space-charge layer $\left(x=x_{w}=2 w-1\right)$ the electric field drops to zero as $E(x) \approx \frac{\sqrt{3}}{2 \pi} \frac{x_{w}-x}{2 w^{2}}\left|\ln \left(x_{w}-x\right)\right|$ whereas the charge density behaves as $\frac{\rho(x)}{2 \epsilon} \approx \frac{\sqrt{3}}{2 \pi} \frac{1}{w}\left(1-\frac{x_{w}-x}{2 w} \ln \left|x_{w}-x\right|\right)$ which is valid on both sides of the edge.

Similar as for the 1D-SCL photo generated current, the 2D-SCL photo generated current (24) is greater than the 1D-SCL injection current (17), in this case $3 \times$. With $K_{\text {phot }}=e g W$ we find in the usual way

$$
\begin{gathered}
W=\left(\frac{6}{\pi} \frac{\mu \epsilon}{e g}\right)^{1 / 3} V^{2 / 3} \\
K_{\text {phot }}=\left(\frac{6}{\pi} \mu \epsilon\right)^{1 / 3}(e g)^{2 / 3} V^{2 / 3}
\end{gathered}
$$

and compared with the $1 \mathrm{D}$ case we obtain a larger voltage dependence $\left(V^{2 / 3}\right.$ instead of $\sqrt{V})$ and a smaller dependence on the irradiance $\left(g^{2 / 3}\right.$ instead of $\left.g^{3 / 4}\right)$.

The saturation voltage is obtained by taking the limit $W \rightarrow L$

$$
V_{\mathrm{sat}}=\sqrt{\frac{\pi}{6} \frac{e g}{\mu \epsilon}} L^{3 / 2}
$$

For $V>V_{\text {sat }}(22)$ is no longer valid since the field is now singular on both sides of the gap. Multiplying (20) with $\left(1-x^{2}\right)$ before integrating now yields

$$
\int_{-1}^{1}\left(1-x^{2}\right) E(x)\left[\int_{-1}^{1} \frac{E\left(x^{\prime}\right)}{x^{\prime}-x} d x^{\prime}\right] d x=-\frac{\beta_{\text {sat }}}{6}
$$

and after reversing the order of integration one finds

$$
\left(\int_{-1}^{1} E(x) d x\right)\left(\int_{-1}^{1} x E(x) d x\right)=\int_{-1}^{1} x E(x) d x=-\frac{\beta_{\text {sat }}}{6}
$$

From (21) with $K_{\text {sat }}=e g L$ we obtain $\beta_{\text {sat }}=3\left(\frac{V_{\text {sat }}}{V}\right)^{2}$ with $0 \leq \beta_{\text {sat }} \leq 3$. The full solution can again be found in the appendix. 


\section{Experimental evidence}

For the 2D-model to be obligatory, two conditions must be fulfilled. First of all the geometry should be two-dimensional, with the thickness of the conductor being much smaller than it's length, and secondly space charge should be present. We considered already two classical cases: injection of a sufficient number of one type of carriers in an insulator or the extraction of photo-generated carriers with non-injecting contacts. If on the contrary one type of carrier can freely flow between the electrodes and the conductor thus behaves ohmically, then the electric field will be uniform but only as long as the accompanying space charge given by (12) is negligible compared with the carrier density. For a conductor with gap length of $10 \mu \mathrm{m}$, an electrode thickness of $100 \mathrm{~nm}, \epsilon=3 \epsilon_{0}$ and a voltage of 10 Volt, the critical density is of the order of $10^{15} \mathrm{~m}^{-2}$, which is small for metallic conductors or semiconductors, but not unusual for an organic conductor. So, even a very thin metallic sheet will behave one-dimensionally and to find evidence for the proposed model we need to look at a relatively poor conductor.

This work is the continuation of previous work we did on the development of a transparent organic photoconductive sensor $[18,28]$, made with typically $80 \mu \mathrm{m}$ wide ITO electrodes, separated by a $20 \mu \mathrm{m}$ gap and covered by a $40 \mathrm{~nm}$ thick hole transporting material (m-MTDAB) and a $20 \mathrm{~nm}$ thick electron transporting material (PTCBI) [18]. Excitons are generated in the PTCBI-layer, diffuse towards the interface and dissociate into an electron in the PTCBI-layer and a hole in the m-MTDAB-layer. The observed current-voltage characteristic under illumination is linear for small voltages but switches to a much smaller slope at some point (see the inset of figure 6 for typical curves). We showed that this is due to a space-charge occurring near the cathode [19]. Using a numerical model [28] we could reproduce the observed behavior using reasonable values for the material parameters and assuming that most of the conduction occurs in the PTCBI-layer, whereas most of the holes remain trapped in the m-MTDAB-layer Contrary to the assumptions made in section 4, and as explained in [28], the fabricated photoconductor has effectively an electron injecting cathode since the active layers covering the cathode form a reverse biased heterojunction (above the anode the heterojunction is forward biased and forms an effective sink for electrons). This explains why there occurs a linear regime without space-charge limitation for small voltages. However the electron current injected from the cathode is limited by the maximal current of the reverse biased diode and when this limit is reached the cathode becomes non-injecting and this causes the sudden change of slope in the characteristics.

Due to the trapping of holes the ideal current-voltage characteristics derived in sections 3 and 4 must be modified. It is well-known that for a 1D model and assuming an exponential distribution of traps, the $J \sim V^{2} / L^{3}$ dependence for the (dark) injection current must be replaced by a dependence $J \sim V^{r+1} / L^{2 r+1}$, where $r=T_{t} / T_{0}$, with $T_{t}$ a temperature describing the trap distribution and $T_{0}$ the ambient temperature. This behavior can be explained on the basis of a 


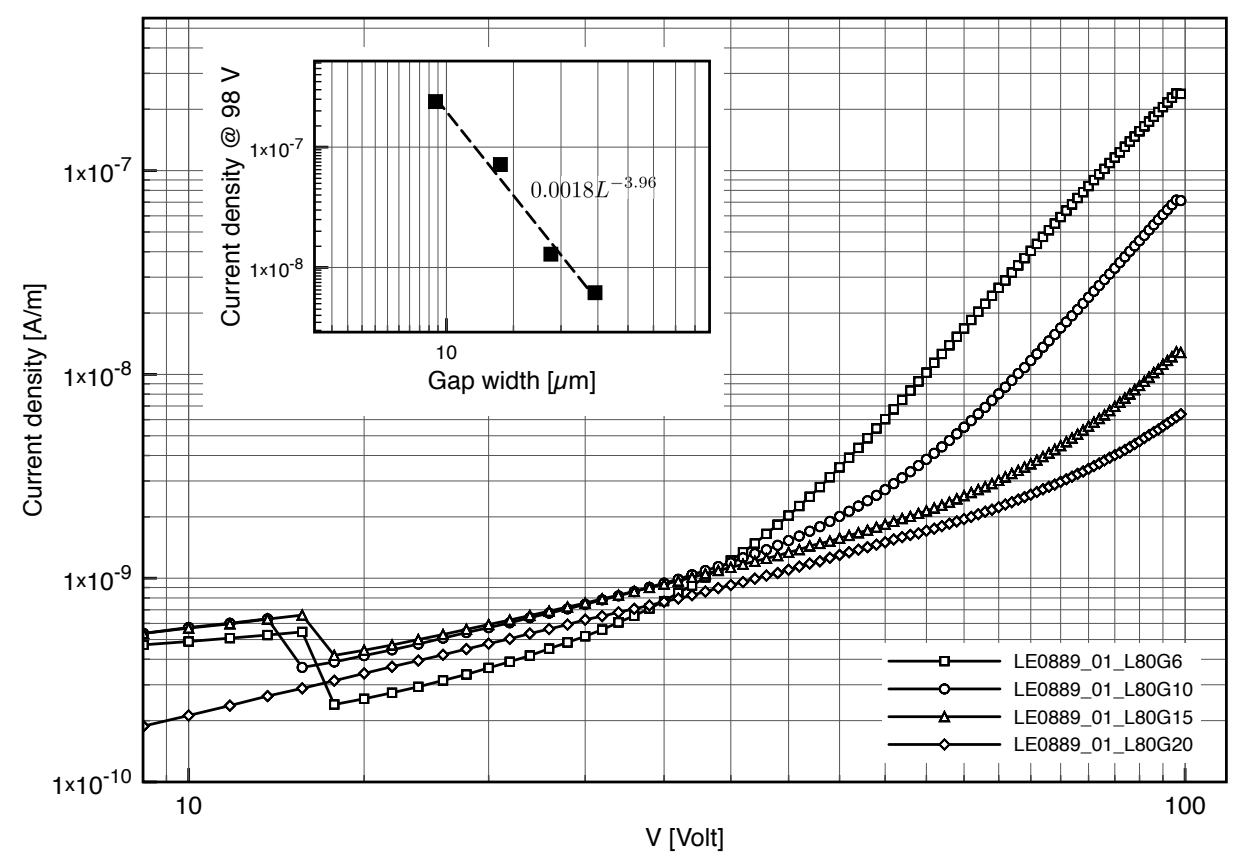

Figure 5: Dark current-voltage characteristics for 4 samples $(20 \mathrm{~nm}$ m-MTDAB $+10 \mathrm{~nm}$ PTCBI) with nominal gap widths in the range 6-20 $\mu \mathrm{m}$. The inset shows the current for $V=98$ Volt as a function of the gap width, which was obtained by measuring it on a single location of the finger pattern. The total length of the electrode fingers is about 1 meter.

model with a small fraction of mobile carriers with fixed mobility whose density depends on the trapped density according to $p \sim p_{t}^{r}$ [8], or considering carriers with a density dependent mobility $\mu \sim p^{r-1}[29,10]$. The dependence can easily be found using a scaling argument as was done by Langmuir [2]. A similar scaling argument can be applied to the equations (12) and (13) of the $2 \mathrm{D}$ model. Assuming uniformity in the direction perpendicular to the film and using e.g. the density dependent mobility model, one finds a dependence $K \sim V^{r+1} / L^{r+1} / D^{r-1}$, with $D$ the thickness of the film. Measurements for relatively thin devices with gap widths in the range 6-20 $\mu \mathrm{m}$ are shown in figure 5 . These measurements resemble those obtained for conventional planar samples, see e.g. [30]. For small voltages the current-voltage relation is linear and for high voltages the currents tend to the same power law $K \sim V^{5.5}$, with $r \approx 4.5$. However, as shown in the inset, the dependence on the gap width $L$ is much smaller than for the 1D conventional device, with a dependence $K \sim L^{-4}$, which is much more in line with the $2 \mathrm{D}$ model. The discrepancy between the observed $L^{-4}$ dependence and the expected $L^{-5.5}$ dependence could be due to the error on the measured gap widths, which have been obtained by measurements on a single location along the meter long finger pattern. 
The effect of trapping on the SCL photo current can be found as before. For the $1 \mathrm{D}$ model $(4)$ is replaced by the more general dependence $J \sim \sqrt{V} g^{\frac{2 r+1}{2 r+2}}$ and for the $2 \mathrm{D}$ model $(26)$ is replaced by $K_{\text {phot }} \sim(g V)^{\frac{r+1}{r+2}}$. For $r=1$ we recover the dependencies without traps. With increasing $r$ the contrast in the voltage dependence increases whereas the contrast in the generation dependence decreases and in both cases tends to a linear dependence. Using $r=4.5$ the $2 \mathrm{D}$-model predicts a $V^{0.85}$ dependence whereas the $1 \mathrm{D}$-model yields a square root dependence independent on the presence of traps. The inset of figure 6 shows typical $I(V)$-characteristics for a range of illuminances and in the dark. For high applied voltages, injection of holes from the anode becomes visible. To compensate for this effect we subtract the dark current from the measured characteristics (shown in broken lines). To compensate for the voltage drop over the neutral region we calculate the voltage over the SCL as $V_{\mathrm{SC}}=V-$ $I / G$, where $G$ is the conductance of the initial linear part of the characteristic (also indicated in the inset). Finally we compensate the current injected from the cathode by subtracting a constant $I_{0}, I_{\mathrm{SC}}=I-I_{0}$. We then obtain the best straight line approximation in a log-log diagram and exclude the low- and high-voltage regions (the intervals used are indicated by a thick line in the inset). For each curve the value of $I_{0}$ was chosen which maximizes the $R^{2}$ value, and these limiting currents are indicated in the inset by the dots. For the highest luminance considered the exponent found $\left(V^{0.85}, R^{2}=0.99951\right)$ fits the 2D-model exactly. For lower luminances the exponent increases, which is probably due to the effect of the injected hole current, which clearly has not been compensated completely.

Although the difference in the predicted dependence on the generation level is small (with $r=4.5$ the $1 \mathrm{D}$ model predicts a $g^{0.91}$ dependence and the $2 \mathrm{D}$ model a slightly lower $g^{0.85}$ dependence), it remains useful to check also this dependence. Data pertaining to the linear regime has been published in [28] and is reproduced in figure 7, augmented with data for the SCL-regime. Data is shown as a function of the irradiance (either a $639.6 \mathrm{~nm}$ laser irradiance or a display backlight luminance converted to an equivalent laser irradiance). The points marked with triangles and squares have been measured for a bias voltage of $0.5 \mathrm{~V}$, which is within the linear regime, whereas the points marked with diamonds show the slope $d I / d V$ for a bias voltage of $10 \mathrm{~V}$, which is in the SCL regime. For the linear regime the triangles show the current $I(V)$, and the squares show the slope of the current $-d I / d t$ just after switching off the irradiance, but with the bias voltage still applied. This quantity enables us to judge the dependence of the generation rate on the irradiance [28]. In the linear regime the generation rate of electron/hole pairs is in equilibrium with the recombination rate and depending on the recombination mechanism this determines the values of the electron and hole densities. For the high irradiance range we interpret the data as evidence for bimolecular recombination and a sublinear dependence of the generation rate on the irradiance [28] $g \sim \Phi^{0.78}$, which could be due to exciton quenching by electrons [31].

In the SCL regime the current increase $d I / d V$ is due to the increase of the 


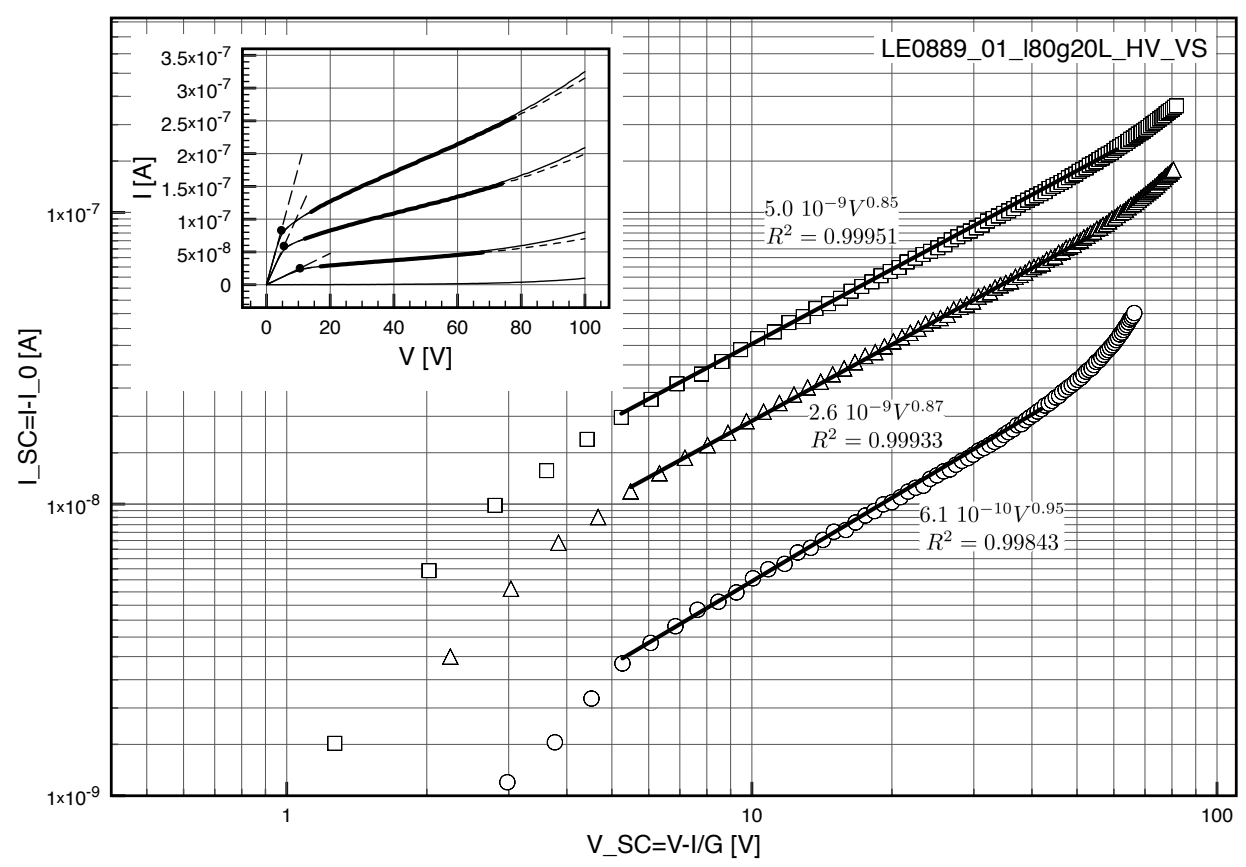

Figure 6: Calculated $I_{\mathrm{SC}}\left(V_{\mathrm{SC}}\right)$-characteristics of the space charge layer of a sample $(20 \mathrm{~nm}$ m-MTDAB $+10 \mathrm{~nm}$ PTCBI) with gap width $20 \mu \mathrm{m}$ and a total electrode length of $2.38 \mathrm{~m}$ on a $\log -\log$ scale. The inset shows the measured $I(V)$-characteristics on a linear scale. Characteristics are shown for 181, 104, 29 and $0 \mathrm{~cd} / \mathrm{m}^{2}$. The thick lines indicate the intervals over which the power law approximations in the main figure have been obtained. 
current egW collected from the space-charge region and the recombination mechanism has no influence.. From the measurements we find a dependence $d I / d V \sim \Phi^{0.61-0.64}$, which can be interpreted as $d I / d V \sim g^{0.78-0.82}$ using again $g \sim \Phi^{0.78}$. This is close to the expected values and confirms that the sublinear dependence of the generation rate on the irradiance could indeed be due to exciton quenching by electrons and not by the electric field.

\section{Conclusions}

Although the current flow in a thin film placed in the gap between two co-planar electrodes is one-dimensional, the electric field in the film must be calculated with a two-dimensional model, at least when space charge effects become important. It was shown that the field can be obtained efficiently using a conformal transformation. By inverting the resulting relation, we found that the spacecharge distribution in the film is equal to the finite Hilbert transform of the in-plane component of the electric field. This enabled us to derive analytical expressions for the electric field and the charge density for several space-charge limited current problems and these expressions were shown to agree very well with numerical calculations.

It was shown that for single carrier injection the one-dimensional Mott-Guerny equation $J=\frac{9}{8} \mu \epsilon \frac{V^{2}}{L^{3}}$ must be replaced by $K=\frac{2}{\pi} \mu \epsilon \frac{V^{2}}{L^{2}}$ for the two-dimensional in-plane layout. Apart from the quadratic dependence on the gap width $L$, the factor $9 / 8$ is replaced by a factor $2 / \pi$. Although this seems not to have been noticed in the past, for space-charge limited photo-carrier extraction the one-dimensional Mott-Guerny equation is actually given by $J=4 \mu \epsilon \frac{V^{2}}{W^{3}}$, where $W$ is the space charge layer width and the characteristic factor $9 / 8$ has been replaced by a factor 4 . For the corresponding two-dimensional layout this is replaced by $K=\frac{6}{\pi} \mu \epsilon \frac{V^{2}}{W^{2}}$. As a result the well-known square root dependence of the space-charge limited current on the applied voltage in the one-dimensional lay-out is replaced by a $V^{1 / 3}$ dependence for the two-dimensional in-plane layout. Similarly the $g^{3 / 4}$ dependence on the generation rate of photo-carriers is replaced by a $g^{2 / 3}$ dependence. Using a scaling argument we derived the voltage and generation dependencies of these currents if trapping is taken into account. The presence of traps increases the contrast between the 1D and 2D models for the voltage dependence but decreases the contrast for the generation dependence. Using measurements of the current-voltage characteristics of a photoconductive sensor with interdigitated electrodes and with an organic bilayer as photo-sensitive material, we showed that the dark injection current and the voltage dependence of the space-charge limited photo current do fit much better with the 2D model than with the 1D model, whereas the generation dependence fits with both, and cannot be used to discriminate between the two models. 


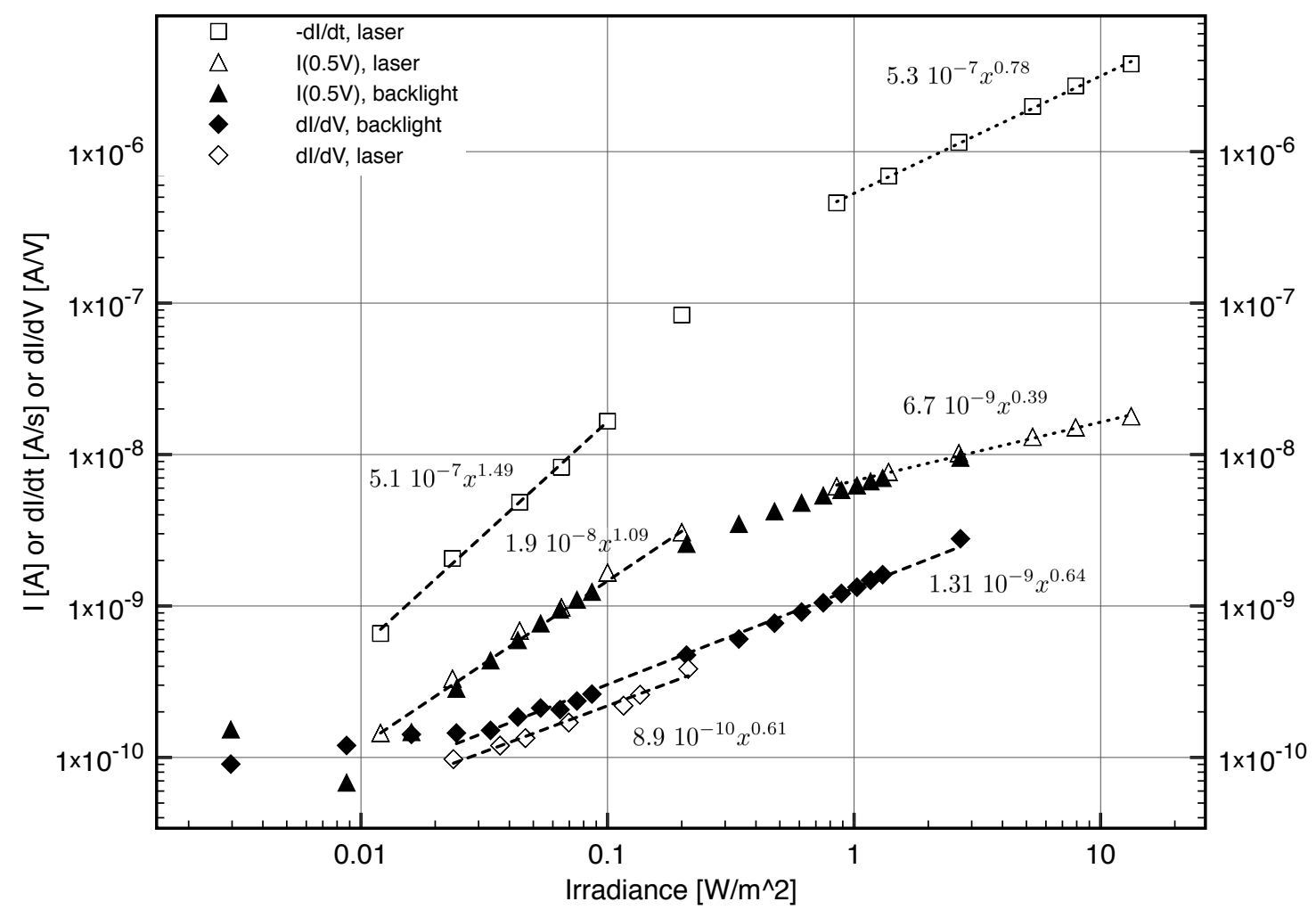

Figure 7: Measurement data for a bilayer in-plane photoconductive sensor as a function of the irradiance. Illumination was either by a $639.6 \mathrm{~nm}$ laser (open symbols) or by a display backlight (closed symbols). In the latter case the luminance was converted to an irradiance according to $2.7 / 373 \mathrm{~W} / \mathrm{cd}$. The triangles show the current in the linear regime, for $V=0.5 \mathrm{~V}$. The squares show the slope of the current decay when switching off the illumination, again for $V=0.5 \mathrm{~V}$. The diamonds show the slope of the $I(V)$ characteristic in the space-charge limited regime, for $V=10 \mathrm{~V}$. 


\section{Acknowledgments}

Part of this work was supported by the TARDIS research \& development project funded by the IWT (Institute for the Promotion of Innovation by Science and Technology in Flanders), and by the Interuniversity Attraction Poles program of the Belgian Science Policy Office, under grant IAP P7-35. 


\section{Bibliography}

[1] C. D. Child, Discharge From Hot CaO, Physical Review 32 (5) (1911) $492-$ 511. doi:10.1103/PhysRevSeriesI.32.492.

URL http://link.aps.org/doi/10.1103/PhysRevSeriesI.32.492

[2] I. Langmuir, The Effect of Space Charge and Residual Gases on Thermionic Currents in High Vacuum, Physical Review 2 (6) (1913) 450-486. doi:10.1103/PhysRev.2.450.

URL http://link.aps.org/doi/10.1103/PhysRev.2.450

[3] M. A. Lampert, P. Mark, Current Injection in Solids, 1st Edition, Electrical Science series, Academic Press, 1970.

[4] P. W. M. Blom, C. Tanase, D. M. de Leeuw, R. Coehoorn, Thickness scaling of the space-charge-limited current in poly(p-phenylene vinylene), Applied Physics Letters 86 (9) (2005) 092105. doi:10.1063/1.1868865.

URL http://scitation . aip.org/content/aip/journal/apl/86/9/10.1063/1.1868865

[5] P. Davids, I. Campbell, D. Smith, Device model for single carrier organic diodes, Journal Of Applied Physics 82 (12) (1997) 6319-6325.

[6] V. Mihailetchi, J. Wildeman, P. Blom, Space-Charge Limited Photocurrent, Physical Review Letters 94 (12) (2005) 126602. doi:10.1103/PhysRevLett.94.126602.

URL http://link.aps.org/doi/10.1103/PhysRevLett.94.126602

[7] J. Shen, J. Yang, Physical mechanisms in double-carrier trap-charge limited transport processes in organic electroluminescent devices: A numerical study, Journal Of Applied Physics 83 (12) (1998) 7706-7714.

URL http://scitation. aip.org/content/aip/journal/jap/83/12/10.1063/1.367942

[8] M. Mandoc, B. de Boer, G. Paasch, P. Blom, Trap-limited electron transport in disordered semiconducting polymers, Physical Review B 75 (19) (2007) 193202. doi:10.1103/PhysRevB.75.193202.

URL http://link.aps.org/doi/10.1103/PhysRevB.75.193202

[9] F. Torricelli, D. Zappa, L. Colalongo, Space-charge-limited current in organic light emitting diodes, Applied Physics Letters 96 (10) (2010) 113304-1050. doi:10.1109/LED.2009.2027998.

URL http://ieeexplore. ieee.org/lpdocs/epic03/wrapper.htm?arnumber $=5229275$

[10] B. Ramachandhran, H. Huizing, R. Coehoorn, Charge transport in metal/semiconductor/metal devices based on organic semiconductors with an exponential density of states, Physical Review B 73 (23) (2006) 233306. doi:10.1103/PhysRevB.73.233306.

URL http://dx.doi.org/10.1103/PhysRevB.73.233306 
[11] Y. Lau, Simple Theory for the Two-Dimensional ChildLangmuir Law, Physical Review Letters 87 (27) (2001) 278301. doi:10.1103/PhysRevLett.87.278301.

URL http://link.aps.org/doi/10.1103/PhysRevLett.87.278301

[12] J. Luginsland, Y. Lau, R. Gilgenbach, Two-Dimensional ChildLangmuir Law, Physical Review Letters 77 (22) (1996) 4668-4670. doi:10.1103/PhysRevLett.77.4668.

URL http://link.aps.org/doi/10.1103/PhysRevLett.77.4668

[13] J. W. Luginsland, Y. Y. Lau, R. J. Umstattd, J. J. Watrous, Beyond the Child-Langmuir law: A review of recent results on multidimensional space-charge-limited flow, Physics Of Plasmas 9 (5) (2002) 2371. doi:10.1063/1.1459453.

URL http://scitation.aip.org/content/aip/journal/pop/9/5/10.1063/1.1459453

[14] S. M. Sze, K. K. Ng, Physics of Semiconductor Devices, 3rd Edition, WileyInterscience, 2007, Ch. 13, pp. 663-742.

[15] A. Kuwahara, S. Naka, H. Okada, Investigation of organic photoconductors, Journal Of Photopolymer Science And Technology 20 (1) (2007) 43-46.

[16] J. C. Ho, A. Arango, V. Bulović, Lateral organic bilayer heterojunction photoconductors, Applied Physics Letters 93 (6) (2008) 063305. doi:10.1063/1.2949317.

[17] J. C. Ho, Organic Lateral Heterojunction Devices for Vapor-phase Chemical Detection, Ph.D. thesis, Massachusetts Institute of Technology (May 2009).

[18] W. Woestenborghs, P. De Visschere, F. Beunis, G. Van Steenberge, K. A. Neyts, A. Vetsuypens, Analysis of a transparent organic photoconductive sensor, Organic Electronics 13 (11) (2012) 2250-2256. doi:10.1016/j.orgel.2012.06.049.

URL http://dx.doi.org/10.1016/j.orgel.2012.06.049

[19] W. Woestenborghs, P. De Visschere, F. Beunis, A. Vetsuypens, K. A. Neyts, Transient and local illumination of an organic photoconductive sensor, in: C. E. Tabor, F. Kajzar, T. Kaino, Y. Koike (Eds.), SPIE OPTO, SPIE, 2013, p. 862216. doi:10.1117/12.2000502.

URL http://proceedings. spiedigitallibrary.org/proceeding. aspx?doi=10.1117/12.2000502

[20] W. Woestenborghs, P. De Visschere, F. Beunis, K. A. Neyts, A. Vetsuypens, Detection of a space-charge region in an organic photoconductive sensor, in: International Display Workshop 2012/Asia Display 2012, J-Global, 2012, pp. 1855-1857.

URL http://jglobal.jst.go.jp/public/20090422/201302296559590666

[21] S. M. Sze, K. K. Ng, Physics of Semiconductor Devices, 3rd Edition, WileyInterscience, 2007, Ch. 6, pp. 293-373. 
[22] C. Lombardo, Z.-E. Ooi, E. Danielson, A. Dodabalapur, Electrical characteristics of lateral organic bulk heterojunction device structures, Organic Electronics 13 (7) (2012) 1185-1191. doi:10.1016/j.orgel.2012.03.003.

URL http://dx.doi.org/10.1016/j.orgel.2012.03.003

[23] Z. E. Ooi, K. L. Chan, C. J. Lombardo, A. Dodabalapur, Analysis of photocurrents in lateral-geometry organic bulk heterojunction devices, Applied Physics Letters 101 (5) (2012) 053301. doi:10.1063/1.4739469.

URL http://scitation.aip.org/content/aip/journal/apl/101/5/10.1063/1.4739469

[24] H. Kober, Dictionary of Conformal Representations, Dover, 1957.

[25] K. Binns, P. Lawrenson, Analysis and Computation of Electric and Magnetic Field Problems, Pergamon Press, 1963.

[26] W. K. Panofsky, M. Philips, Classical Electricity and Magnetism, 2nd Edition, Addison-Wesley series in physics, Addison-Wesley Publishing Company, Inc., 1962.

[27] F. Tricomi, Integral equations, Dover, 1985, Ch. 4, pp. 173-185.

[28] W. Woestenborghs, P. D. Visschere, F. Beunis, K. Neyts, A twodimensional model for an in-plane organic photo-conductive bilayer sensor, Journal of Physics D: Applied Physics 47 (35) (2014) 355103. doi:10.1088/0022-3727/47/35/355103.

URL http://stacks.iop.org/0022-3727/47/i=35/a=355103

[29] M. C. J. M. Vissenberg, Theory of the field-effect mobility in amorphous organic transistors, Physical Review B 57 (20) (1998) 12964-12967. doi:10.1103/PhysRevB.57.12964.

URL http://dx.doi.org/10.1103/PhysRevB.57.12964

[30] P. W. M. Blom, M. deJong, J. Vleggaar, Electron and hole transport in poly(p-phenylene vinylene) devices, Applied Physics Letters 68 (23) (1996) 3308-3310.

URL http://scitation.aip.org/content/aip/journal/apl/68/23/10.1063/1.116583

[31] Y. Luo, H. Aziz, G. Xu, Z. D. Popovic, Electron-induced quenching of excitons in luminescent materials, Chemistry Of Materials 19 (9) (2007) 2288-2291. doi:10.1021/cm062105s.

[32] A. S. Peters, The Solution of Some Non-Linear Integral Equations with Cauchy Kernels, Tech. Rep. IMM-NYU 307 (Jan. 1963).

URL https://archive.org/details/solutionof someno00pete 


\section{AppendixA. Solution of the non-linear integral equation}

The equation obtained in the main text for the electric field (15) or (20) has the general form

$$
\lambda \phi(x)+\phi(x) \int_{-1}^{1} \frac{\phi\left(x^{\prime}\right)}{x^{\prime}-x} d x^{\prime}=f(x)
$$

where the constant $\lambda$ and the function $f(x)$ are known. For the examples considered $\lambda=0 . \quad \lambda \neq 0$ is obtained e.g. for single carrier injection in a medium with a background carrier density being present. In that case we can observe the transition from ohmic conduction to space-charge limited conduction. A detailed solution of this equation has been published by Peters [32]. To obtain the solution one introduces the following function defined in the complex $z$-plane

$S(z)=\lambda+\int_{-1}^{1} \frac{\phi(t)}{t-z} d t=\left[\frac{2}{1-z^{2}} \int_{-1}^{1} \frac{\left(1-t^{2}\right) f(t)}{t-z} d t+\frac{2 \lambda\left(k_{1}+k_{0} z\right)-k_{0}^{2}}{1-z^{2}}+\lambda^{2}\right]^{1 / 2}$

where $k_{0}=\int_{-1}^{1} \phi(x) d x$ and $k_{1}=\int_{-1}^{1} x \phi(x) d x$ are the first two moments of the unknown function. Peters shows that for (A.1) to have a solution, these constants should be chosen so that $S(z)$ is analytic in the complex plane outside the cut $(-1,1)$. Usually this condition is not sufficient to fix $k_{0}$ and $k_{1}$ and additional boundary conditions are needed, as illustrated in the main text. The electric field and the charge density for $-1 \leq x \leq 1$ can then be found by taking the limit $z \rightarrow x \pm j 0$

$$
\begin{aligned}
\phi(x) & =\frac{1}{\pi} \Im[S(x+j 0)] \\
\frac{1}{\pi} \int_{-1}^{1} \frac{\phi(t)}{t-x} d t & =\frac{1}{\pi} \Re[S(x+j 0)]-\frac{\lambda}{\pi}
\end{aligned}
$$

where

$S(x+j 0)=\left[\frac{2}{1-x^{2}} \int_{-1}^{1} \frac{\left(1-t^{2}\right) f(t)}{t-x} d t+\frac{2 \lambda\left(k_{1}+k_{0} x\right)-k_{0}^{2}}{1-x^{2}}+\lambda^{2}+2 j \pi f(x)\right]^{1 / 2}$

For single carrier injection into an insulator the electric field follows by solving (15) and thus $\lambda=0$ and $f(x)=\alpha / 4$. One obtains

$$
S_{\mathrm{inj}}(x+j 0)=\left[\frac{\alpha}{2} \ln \frac{1-x}{1+x}-\frac{k_{0}^{2}+\alpha x}{1-x^{2}}+j \frac{\pi}{2} \alpha\right]^{1 / 2}
$$

Since we did prescribe the potential, $k_{0}=1$ and $\alpha$ is the new unknown in this case. By exploiting the boundary condition $E(-1)=0$ we found (16), thus $\alpha=1$. This can also be seen from (A.6) where the choice $\alpha=1$ removes the singularity for $x=-1$ and we obtain

$$
S_{\mathrm{inj}}(x+j 0)=\left[\frac{1}{2} \ln \frac{1-x}{1+x}-\frac{1}{1-x}+j \frac{\pi}{2}\right]^{1 / 2}
$$


For photo-carrier extraction (20) must be solved with again $\lambda=0$ but with a linear dependence for $f(x)$

$$
f(x)=\frac{\beta}{8} \begin{cases}\left(x-x_{w}\right) & -1<x<x_{w} \\ 0 & x_{w}<x<1\end{cases}
$$

where $x_{w}=2 w-1$ is the position of the edge of the space-charge layer. Inserting this into (A.5), with $k_{0}=1$ one obtains after some calculations (and for $-1<$ $\left.x<x_{w}\right)$

$$
\begin{aligned}
S_{\text {phot }}^{2}(x+j 0)=\frac{\beta}{4}\left(x-x_{w}\right) \ln \frac{x_{w}-x}{1+x}+\frac{\beta}{4} w \frac{1-x_{w}+2 x}{1+x} & \\
& +\frac{\frac{\beta}{3} w^{3}-1}{1-x^{2}}-j \frac{\pi}{4} \beta\left(x_{w}-x\right) \quad x<x_{w}
\end{aligned}
$$

Outside the space-charge layer we obtain similarly

$$
S_{\text {phot }}^{2}(x+j 0)=\frac{\beta}{4}\left(x-x_{w}\right) \ln \frac{x-x_{w}}{x+1}+\frac{\beta}{4} w \frac{2 x-x_{w}+1}{x+1}+\frac{\frac{\beta}{3} w^{3}-1}{1-x^{2}} \quad x>x_{w}
$$

If $w<1$, then there is no singularity for $x=x_{w}$ and $\beta$ has been obtained in (23) $\left(\beta w^{3}=3\right)$ by removing the remaining singularity for $x=-1$. Note that (i) since $S_{\text {phot }}^{2}\left(x_{w}\right)=\beta w / 4>0, E\left(x_{w}\right)=0$; and (ii) although $E(x)=0$ for $x_{w} \leq x<1, \rho(x) \neq 0$ and the choice $\beta w^{3}=3$ avoids a singularity in $\rho(x)$ for $x=1$. The final expressions are then

$$
\begin{gathered}
S_{\text {phot }}(x+j 0)=\sqrt{\frac{3}{4 w^{3}}}\left[\left(x-x_{w}\right) \ln \frac{x_{w}-x}{1+x}+w \frac{1-x_{w}+2 x}{1+x}-j \pi\left(x_{w}-x\right)\right]_{(\mathrm{A} .11)}^{1 / 2} x<x_{w} \\
S_{\text {phot }}(x+j 0)=\sqrt{\frac{3}{4 w^{3}}}\left[\left(x-x_{w}\right) \ln \frac{x-x_{w}}{x+1}+w \frac{2 x-x_{w}+1}{x+1}\right]^{1 / 2} x>x_{w}
\end{gathered}
$$

If saturation occurs $(w=1)$ we obtain

$$
S_{\text {phot }}^{2}(x+j 0)=\frac{\beta_{\text {sat }}}{4}(x-1) \ln \frac{1-x}{1+x}+\frac{\beta_{\mathrm{sat}}}{4} \frac{2 x}{1+x}+\frac{\frac{\beta_{\mathrm{sat}}}{3}-1}{1-x^{2}}-j \frac{\pi}{4} \beta_{\mathrm{sat}}(1-x)
$$

Singularities occur on both sides and different values for $\beta_{\text {sat }}$ in the range $3 \geq$ $\beta_{\text {sat }}>0$ correspond with different voltages $V_{\text {sat }} \leq V<\infty$. 